\title{
Anelpe Onegenal
}

\section{COMPORTAMENTO SEXUAL DE RISCO ENTRE ESTUDANTES UNIVERSITÁRIAS DOS CURSOS DE CIÊNCIAS DA SAÚDE}

Angela Maria Moser*, Claudete Reggiani, Almir Urbanetz

Trabalho realizado no Departamento de Tocoginecologia, Programa de Pós-Graduação em Tocoginecologia, Setor de Ciências da Saúde da Universidade Federal do Paraná

*Correspondência:

Rua Rio Branco, 1250

Medianeira/PR

Cep: $85884-000$

Fone/fax: (45) 3264-3330

angelamoser@arnet.com.br

\begin{abstract}
RESUMO
Objetivo. Avaliar o comportamento sexual de estudantes do sexo feminino do Setor de Ciências da Saúde da Universidade Federal do Paraná e do Setor de Ciências Biológicas e da Saúde da Universidade Estadual do Oeste do Paraná, Campus Cascavel. Métodos. Participaram todas as estudantes matriculadas nos referidos setores, no mês de junho de 2001 , com idade entre 18 e 24 anos completos, sendo que a amostra final foi constituída por 572 estudantes em Curitiba e 395 em Cascavel. Foram avaliados a idade, relacionamento famillar, religiosidade, participação em aulas de educação sexual, idade de início de atividade sexual, uso de métodos anticoncepcionais e preservativos, e número de parceiros para avaliar a conduta sexual. Sexo seguro foi definido pelo uso de preservativo em todas ou na maioria das relações sexuais pelas estudantes monogâmicas, incluindo as estudantes poligâmicas com uso de preservativo em todas as relações sexuais. Sexo inseguro foi definido pelo uso ocasional de preservativo pelas estudantes monogâmicas, e pelo não uso sistemático entre as estudantes poligâmicas. O instrumento de coleta de dados foi um questionário auto-respondido. A análise dos dados foi feita no SPSS, utilizando o teste do Quiquadrado de Pearson e Yates, teste do p de Wilcoxon-Gehan, análise bivariada e regressão logística.

Resultados. Não houve diferença siggificativa na conduta sexual de risco entre as estudantes e a cidade de estudo. Cerca de $50 \%$ das estudantes de 18 a 20 anos e $70 \%$ das de 21 a 24 anos tinham vida sexual ativa. A abstinência esteve associada a menor idade, maior freqüência a culto religioso e ao bom relacionamento das estudantes com os pais. A prática de sexo seguro esteve diretamente associada a menor idade e a morar fora da residência da família. Ter participado de aulas de educação sexual esteve associada a maior abstinência, mas não a sexo seguro, e a relaçãa não se manteve na análise multivariada.

Conclusão. Relacionamento familiar saudável e religiosidade estão associados à conduta de sexo seguro. É relevante a percentagem de estudantes que ainda têm uma conduta de sexo inseguro, mostrando que ser universitária e freqüentar cursos de ciências da saúde não são garantia de comportamento sexual seguro.
\end{abstract}

Unitermos: Comportamento sexual. Abstinência sexual. Sexo seguro.

\section{INTRODUÇÃO}

O termo adolescer tem suas raízes na civilização greco-romana que empregava o verbo adolere para designar arder, queimar. Este verbo inclui o prefixo ad, que indica direção (em direção a). Dolere é a raiz da palavra, que se traduz por sofrer uma dor, lamentar, estar triste. Então adolescer significa ir em direção à dor, ou ir em direção ao que arde, que queima!

Os limites cronológicos da adolescência são definidos pela Organização Mundial da Saúde (OMS) entre 10 e 19 anos (adolescents) e pela Organização das Nações Unidas (ONU) entre 15 e 24 anos (youth). Utiliza-se ainda o termo jovens adultos para a faixa etária de 20 a 24 anos de idade (young adults). Nas normas e políticas de saúde do Ministério da Saúde do Brasil, os limites da faixa etária de interesse são as idades de 10 a 24 anos². $^{2}$.

No modelo sociocultural atual, o conflito que se torna mais evidente neste processo de mudança é aquele relacionado com a sexualidade das adolescentes. E, na busca do exercício pleno desta sexualidade, pode ocorrer o início precoce da atividade sexual, com orientação inadequada ou ausente a respeito de métodos contraceptivos e prevenção de doenças sexualmente transmissíveis/síndrome da imunodeficiência adquirida (DST/aids), o que tem levado ao crescente número de gravidezes indesejadas e abortamentos provocados, com repercussões emocionais, orgânicas e socioeconômicas importantes. Existem pesquisas que mostram que as adolescentes que iniciam precocemente a atividade sexual terão um maior número de parceiros sexuais e, portanto, um maior risco de adquirir DST, o que poderá não somente influenciar na sua fertilidade, mas também aumentar a suscetibilidade para adquirir uma infecção pelo HIV (vírus da imunodeficiência adquirida) $)^{3,4}$.

O Boletim Epidemiológico AIDS5 ${ }^{5}$ do Ministério da Saúde, mostra os casos de aids em jovens do sexo feminino com 19 anos de idade ou mais, segundo a escolaridade. Em 1991, 10\% eram jovens com 8 a II anos de escolaridade e 5,3\% com 12 anos ou mais. No ano de 2002, aumentaram os casos na escolaridade de 8 
a 1 anos: 18,4\%, mas diminuíram naquelas com 12 anos ou mais: $4,9 \%$. Estes dados indicam que os níveis de educação e informação podem ter forte influência na queda de ocorrência de aids.

Pouco se sabe sobre a conduta sexual de jovens do sexo feminino que estudam nas universidades brasileiras e, menos ainda, no Estado do Paraná. Teoricamente, por possuírem um nível de informação melhor, deveriam ter conduta de menor risco que adolescentes e jovens com menor educação. Ademais, é possível que existam diferenças no exercício da sexualidade entre estudantes universitárias que moram na capital, quando comparadas com aquelas que moram em cidade do interior do Estado.

Esta pesquisa se propôs a estudar o comportamento sexual de estudantes do sexo feminino do Setor de Ciências da Saúde da Universidade Federal do Paraná em Curitiba; e do Setor de Ciências Biológicas e da Saúde da Universidade Estadual do Oeste do Paraná, Campus Cascavel.

Cascavel, município situado no extremo oeste do Paraná, tem a população de 256.390 habitantes; e, no ano de 2001, 21,5\% dos nascidos vivos eram filhos de adolescentes. Curitiba, capital do Estado, tem I.646.644 habitantes e também, no ano de 200I, $19 \%$ de nascidos vivos eram filhos de adolescentes ${ }^{6}$.

Será que o conhecimento adquirido em cursos nos quais, ao menos teoricamente, a saúde do ser humano é a principal meta estaria sendo utilizado para uma vida sexual sem riscos de adquirir uma DST/ aids ou uma gravidez não planejada? Será que existe diferença no comportamento sexual entre estudantes da capital e de cidade do interior? Será que a família, religião, o local de moradia têm realmente influência na conduta sexual? Será que existe associação entre a orientação sexual recebida e a conduta sexual das estudantes?

Este trabalho foi realizado para conhecer o comportamento sexual de uma população de estudantes universitárias, o que é de particular interesse por se tratarem de futuras profissionais que, em poucos anos, estarão trabalhando com crianças e outros adolescentes, servindo de modelo e propagando conhecimentos.

\section{Métodos}

Este é um estudo de corte transversal, observacional e analítico, realizado em Curitiba e Cascavel (município de médio porte localizado na região oeste do Paraná), no mês de junho de 200 l.

Foram incluídas estudantes da graduação de 18 a 24 anos, de sexo feminino, dos cursos do Setor de Ciências da Saúde da UFPR, e dos cursos do Setor de Ciências Biológicas e da Saúde da Unioeste, Campus Cascavel, que aceitaram voluntariamente participar da pesquisa.

Em Curitiba, o número de estudantes do sexo feminino matriculadas nos cursos a serem estudados era de 1076. Foram aplicados 7/4 questionários entre as estudantes que estavam em sala de aula, sendo que 84 foram devolvidos em branco (não desejo de participar), e 58 excluídos em razão da idade.

Em Cascavel, eram 608 estudantes matriculadas. Foram aplicados 446 questionários entre as estudantes que estavam em sala de aula, sendo que apenas três foram devolvidos em branco (não desejo de participar) e, 48 excluídos em razão da idade.
A amostra final foi constituída por 572 estudantes em Curitiba e 395 em Cascavel.

O instrumento de coleta de dados utilizado foi um questionário auto-respondido, contendo 22 perguntas, sendo que nove questões deveriam ser escritas e as restantes eram de múltipla escolha. O questionário foi pré-testado entre estudantes do terceiro período da Faculdade Tecnológica Ambiental do CEFET-Unidade Medianeira. A coleta dos dados foi realizada na sala de aula das estudantes pela pesquisadora principal, que explicou os objetivos da pesquisa, a importância da participação das estudantes, assim como o direito da não participação e garantiu confidencialidade e a privacidade das informações recebidas.

Para este trabalho foram analisados especificamente os dados referentes à conduta sexual de risco para a contaminação por DST/ aids, utilizando as variáveis independentes: local da universidade, idade, relacionamento familiar, religião, pessoa com quem a estudante morava na época da entrevista, educação sexual pregressa e percepção da estudante sobre a eficácia destas aulas.

Conduta sexual foi definida como o comportamento sexual nos últimos doze meses, utilizando as variáveis dependentes: existência ou não de atividade sexual, número de parceiros, uso de preservativo e freqüência, com parceiro fixo e/ou ocasional. Foram classificados três tipos de conduta sexual:

- Abstinência: estudantes que nunca tiveram relação sexual ou aquelas que não tiveram parceiro nos últimos 12 meses.

- Sexo seguro: estudantes monogâmicas e que referiram usar preservativo "todas as vezes" ou "quase todas as vezes"; também inclui estudantes poligâmicas que afirmaram usar preservativo "todas as vezes" com parceiro fixo e/ ou ocasional.

- Sexo inseguro: estudantes monogâmicas que responderam usar preservativo "de vez em quando" ou "nunca"; estudantes poligâmicas que não responderam usar preservativo "todas as vezes" com parceiros fixos ou ocasionais.

Criou-se a estrutura do banco de dados no Data Entry do SPSS (Statistical Package for the Social Sciences). Em seguida, procedeu-se a entrada dos dados, com digitação dupla (por dois digitadores diferentes). Após a digitação, fez-se a consistência lógica do banco de dados. A análise dos dados foi feita no mesmo SPSS e foram utilizados o teste de Qui-quadrado de Pearson e Yates, sendo que o nível de significância p foi de 5\%. Realizou-se ainda análise múltipla por regressão logística.

O projeto de pesquisa foi aprovado em reunião plenária departamental da Tocoginecologia em 08/05/2001 e depois registrado no Banpesq sob o número 2001009046. Após este procedimento, foi encaminhado ao Comitê de Ética em Pesquisa em Seres Humanos do Hospital de Clínicas/Universidade Federal do Paraná, sendo aprovado na reunião do dia 29/05/2001, estando de acordo com as normas éticas estabelecidas pela Resolução n 196/96 do Ministério da Saúde. Foi emitido o parecer de que não seria necessário o Termo de Consentimento Livre e Esclarecido, mas apenas o preenchimento de um protocolo pelas estudantes, aceitando ou não participar da pesquisa. 


\section{Resultados}

Cerca de $60 \%$ das estudantes declararam ter atividade sexual nos últimos 12 meses. Essa porcentagem desce para 49,2\% entre aquelas de 18 a 20 anos e sobe a $73 \%$ no grupo de 21 a 24 anos. Houve uma associação significativa $(p=0,040)$ entre o tipo de relacionamento entre os pais e atividade sexual, sendo que a atividade sexual foi em torno de 45 pontos percentuais mais freqüente entre as filhas de pais separados. Assim também, o tipo de relacionamento entre a estudante e os pais determinou uma associação significativa $(p=0,0002)$, sendo a abstinência sexual 18 pontos percentuais maior entre as estudantes que declararam "sensação de serem amadas" (Tabela I).

Quanto maior a freqüência ao culto religioso, menor foi a porcentagem de estudantes com atividade sexual, de forma que as que freqüentavam quatro vezes ou mais por mês declararam ser sexualmente ativas em proporção 27 pontos percentuais menor que aquelas que nunca freqüentavam culto religioso.

Houve associação entre atividade sexual e morar atualmente com a família ou sozinha.

A pessoa com quem a estudante residia determinou diferença significativa na conduta sexual $(p=0,035)$. Praticamente não houve diferença quanto à abstinência, mas a prática de sexo inseguro foi maior entre as estudantes que moravam com os pais ou parceiros (34\%) do que entre aquelas que moravam sozinhas ou com outra pessoa $(26,6 \%)$.

A proporção de estudantes com atividade sexual foi significativamente maior entre aquelas que participaram em aulas de educação sexual em alguma etapa de sua vida $(p=0,025)$, porém não houve a mesma associação com a percepção das estudantes da eficácia da educação sexual recebida.

Tabela I - Distribuição percentual das estudantes segundo a conduta sexual nos últimos I2 meses e variáveis sociodemográficas e comportamentais, pessoa com quem mora, participação em aulas de educação sexual e eficácia da orientação

\begin{tabular}{|c|c|c|c|c|c|}
\hline \multirow[b]{2}{*}{ Variável } & \multicolumn{3}{|c|}{ Conduta sexual } & \multirow[b]{2}{*}{$\mathrm{n}$} & \multirow[b]{2}{*}{$\mathbf{p}^{*}$} \\
\hline & Abstinência & Sexo seguro & Sexo inseguro & & \\
\hline Cidade & & & & & 0,554 \\
\hline Curitiba & 39,2 & 28,8 & 32,0 & (569) & \\
\hline Cascavel & 40,4 & 30,8 & 28,8 & (386) & \\
\hline Idade (anos) & & & & & $<0,00 \mid$ \\
\hline $\begin{array}{l}18-20 \\
21-24\end{array}$ & $\begin{array}{l}50,8 \\
27,0\end{array}$ & $\begin{array}{l}26,5 \\
33,3\end{array}$ & $\begin{array}{l}22,7 \\
39,8\end{array}$ & $\begin{array}{l}(510) \\
(445)\end{array}$ & \\
\hline Como é (era) o relacionamento entre os pais $\#$ & & & & & 0,040 \\
\hline Convivência em ambiente de amor e carinho & 41,7 & 29,7 & 28,6 & (734) & \\
\hline Convivência em ambiente de brigas & 40,8 & 25,5 & 33,7 & (98) & \\
\hline Pais separados & 27,1 & 33,6 & 39,3 & $(107)$ & \\
\hline Como é o relacionamento entre a estudante e os pais & & & & & 0,0002 \\
\hline Sensação de ser amada & 41,0 & 29,5 & 29,5 & (847) & \\
\hline Sensação de ser aceita/indiferenç̧a ou sensação de rejeição & 28,9 & 29,9 & 41,2 & (97) & \\
\hline Freqüência a culto religioso ( $n^{0}$ de vezes/mês) & & & & & $<0,00 \mid$ \\
\hline Nenhuma & 31,2 & 30,1 & 38,8 & (276) & \\
\hline | & 32,8 & 33,3 & 33,9 & (189) & \\
\hline 2 & 40,2 & 40,2 & 19,5 & $(87)^{\prime}$ & \\
\hline 3 & 47,2 & 33,3 & 19,4 & $(72)$ & \\
\hline$\geq 4$ & 54,3 & 21,7 & 24,0 & (254) & \\
\hline Pessoa com quem mora & & & & & 0,035 \\
\hline Pais e/ou parceiro & 38,8 & 27,2 & 34,0 & $(518)$ & \\
\hline Sozinha ou com outra pessoa & 40,8 & 32,6 & 26,6 & $(436)$ & \\
\hline Participação em aulas de educação sexual & & & & & 0,025 \\
\hline Sim & 42,0 & 27,8 & 30,2 & $(719)$ & \\
\hline Não & 32,6 & 35,2 & 32,2 & (236) & \\
\hline Eficácia da orientação & & & & & 0,275 \\
\hline Nuncateve & 32,6 & 35,2 & 32,2 & (236) & \\
\hline Insuficiente & 38,3 & 30,2 & 31,5 & (149) & \\
\hline Satisfatória & 40,7 & 27,1 & 32,2 & (432) & \\
\hline Grande eficácia & 35,8 & 35,8 & 28,3 & (106) & \\
\hline
\end{tabular}

*Teste Qui-quadrado de Pearson

\#Foram excluídas as filhas de mãe solteira 
$\mathrm{Na}$ análise por regressão logística, a menor idade, a maior freqüência a culto religioso e o bom relacionamento com os pais mantiveram uma relação positiva com abstinência sexual.

Ao analisar somente as estudantes que declararam ser sexualmente ativas nos últimos 12 meses, foi observado que elas se dividiam em partes quase iguais entre as que praticavam sexo seguro $(49,1 \%)$ e as que declararam sexo inseguro $(50,9 \%)$ (Tabela 2).

Não houve associação da prática de sexo seguro com cidade onde estudavam, qualidade do relacionamento entre os pais e da estudante com os pais, participação em aulas de educação sexual e percepção da eficácia desta orientação. Por outra parte, o sexo seguro era praticado por uma proporção 10 pontos percentuais mais elevada de estudantes que moravam sozinhas em comparação com as que moravam com sua família (pais, parceiro) $(p=0,0 \mid 5)$, e oito pontos percentuais mais alta nas estudantes de até 20 anos do que entre as de mais idade $(p=0,06)$.

Também se observou uma associação, bem mais complexa, entre freqüência de culto religioso e sexo seguro $(p=0,016)$. A menor proporção de estudantes que praticavam sexo seguro observou-se entre aquelas que nunca assistiam a culto religioso. No entanto, as estudantes que mais participam de culto religioso praticavam sexo seguro em proporção 20 pontos percentuais inferior àquelas que assistiam duas vezes por mês. Em outras palavras, a relação entre sexo seguro e religiosidade, avaliada por meio da freqüência ao culto, mostra que a menor prática de sexo seguro se observa em ambos os extremos, ou seja, entre aquelas que nunca freqüentam e as que mais freqüentam culto religioso.

Após a análise por regressão logística, observou-se a manutenção da associação entre abstinência sexual e as estudantes de menor idade $(p<0,00 \mathrm{I})$, maior freqüência a culto religioso $(p<0,00 \mathrm{I})$ e bom relacionamento com os pais $(p=0,016)$. As variáveis que estiveram associadas à conduta de sexo seguro nos últimos 12 meses foram a menor idade $(p=0,002)$ e o fato de morarem sozinhas ou com outras pessoas que não a família $(p=0,017)$.

\section{Discussão}

A observação de que a metade das estudantes universitárias de 18 a 20 anos de cursos de ciências da saúde era sexualmente ativa, percentagem que passou a $70 \%$ entre as estudantes de 21 a 24 anos, corresponde aproximadamente ao que se observa em outros estudos $3,7,8,9,10$.

Também não é surpresa encontrar-se maior porcentagem de abstinência sexual entre as que freqüentavam culto religioso se considerarmos esta freqüência como indicador de aderência à religião, que em geral promove a abstinência sexual". Em relação à religiosidade, os dados são semelhantes aos encontrados entre estudantes de medicina e enfermagem na Austrália'2; os que relatavam freqüência de três ou mais vezes por mês a culto religioso tinham um menor número de parceiros, enquanto na presente pesquisa a mesma freqüência é associada a uma maior prática de abstinência sexual. Vale lembrar, entretanto, que ainda entre aquelas que relataram frequência de três vezes ou mais por mês ao culto, perto de $50 \%$ tinham atividade sexual.

\begin{tabular}{|c|c|c|c|c|}
\hline Variável & $\begin{array}{l}\text { Sexo } \\
\text { seguro }\end{array}$ & $\begin{array}{c}\text { Sexo } \\
\text { inseguro }\end{array}$ & $\mathrm{n}$ & $p^{*}$ \\
\hline Cidade & & & & $0,350+$ \\
\hline Curritiba & 47,4 & 52,6 & (346) & \\
\hline Cascavel & 51,7 & 48,3 & (230) & \\
\hline Idade (anos) & & & & $0,060+$ \\
\hline $\begin{array}{l}18-20 \\
21-24\end{array}$ & $\begin{array}{l}53,8 \\
45,5\end{array}$ & $\begin{array}{l}46,2 \\
54,5\end{array}$ & $\begin{array}{l}(25 I) \\
(325)\end{array}$ & \\
\hline $\begin{array}{l}\text { Como é (era) o relacionamento } \\
\text { entre os pais }{ }^{\#}\end{array}$ & & & & $0,438 *$ \\
\hline Convivência em ambiente de amor e carinho & 50,9 & 49,1 & (428) & \\
\hline Convivência em ambiente de brigas & 43,1 & 56,9 & (58) & \\
\hline Paisseparados & 46,2 & 53,8 & (78) & \\
\hline $\begin{array}{l}\text { Como é o relacionamento entre } \\
\text { a estudante e os pais }\end{array}$ & & & & $0,457 *$ \\
\hline Sensação de seramada & 50,0 & 50,0 & (502) & \\
\hline Sensação de ser aceita & 41,5 & 58,5 & (53) & \\
\hline Indiferença ou sensação de rejeição & 43,8 & 56,3 & $(16)$ & \\
\hline $\begin{array}{l}\text { Freqüência a culto religioso } \\
\text { (nº de vezes/mês) }\end{array}$ & & & & $0,016 *$ \\
\hline Nenhuma & 43,7 & 56,3 & $(190)$ & \\
\hline | & 49,6 & 50,4 & (I27) & \\
\hline 2 & 67,3 & 32,7 & $(52)$ & \\
\hline 3 & 63,2 & 36,8 & (38) & \\
\hline$\geq 4$ & 47,4 & 52,6 & $(\mid 16)$ & \\
\hline Pessoa com quem mora & & & & $0,015+$ \\
\hline Pais e/ou parceiro & 44,5 & 55,5 & $(317)$ & \\
\hline Sozinha ou com outra pessoa & 55,0 & 45,0 & (258) & \\
\hline $\begin{array}{l}\text { Participação em aulas } \\
\text { de educação sexual }\end{array}$ & & & & $0,4 \mid 4+$ \\
\hline Sim & 48,0 & 52,0 & $(4 \mid 7)$ & \\
\hline Não & 52,2 & 47,8 & (159) & \\
\hline Eficácia da orientação & & & & $0,385 *$ \\
\hline Nuncateve & 52,2 & 47,8 & $(159)$ & \\
\hline Insuficiente & 48,9 & 51,1 & (92) & \\
\hline Satisfatória & 45,7 & 54,3 & (256) & \\
\hline Grande eficácia & 55,9 & 44,1 & (68) & \\
\hline
\end{tabular}

+TesteQui-quadradode Yates

*TesteQui-quadradodePearson

\#Foramexclúidasasfihlasdemãesolteira

Um achado interessante foi a associação entre abstinência sexual e sensação de sentir-se amada pelos pais. É possível especular que aquelas que não têm esse sentimento por parte dos pais tenham maior tendência a procurar o amor fora da família e, como conseqüência, maior possibilidade de estabelecer relacionamento com seu parceiro que inclua a prática do sexo. Por outra parte, o relacionamento familiar turbulento, com rejeição e separação entre os pais, está mais relacionado com maior proporção de estudantes com atividade sexual, o que também foi encontrado em uma 
Moser AM ET AL.

pesquisa com adolescentes de segundo grau de São Paulo ${ }^{13}$. Entretanto, esta associação desapareceu na análise multivariada, provavelmente porque deve estar associada à percepção de ser amada ou rejeitada pelos pais, que mostrou mais forte associação com abstinência sexual. A desavença dentro de casa talvez possa ser o fator que leve a adolescente a procurar num parceiro a estabilidade emocional que não the foi oferecida na sua família, o que também foi mostrado por Karofsky'4.

A maior proporção de estudantes que relataram abstinência entre as que participaram de atividades de educação sexual que entre as que não participaram é encorajadora e vai contra o temor expressado por alguns de que a educação em sexualidade seja um estímulo ao início precoce da atividade sexual. Estes resultados estão de acordo com os de diversos pesquisadores que referem mudanças no comportamento sexual após programas bem orientados, principalmente no que diz respeito à prevenção de DST/aids, entre universitários ${ }^{15-17}$.

É verdade que esta associação não se manteve ao realizar a análise multivariada, mas pelo menos é forte indicação de que, no mínimo, a educação em sexualidade não é um estímulo para a prática sexual, confirmando estudos que mostram que programas de educação em sexualidade que cumprem com determinados requisitos, longe de estimular, levam a um exercício responsável da sexualidade ${ }^{18}$.

A tentativa de avaliar a qualidade da educação sexual recebida pelas estudantes que participaram deste estudo por meio de sua percepção da eficácia foi evidentemente pouco discriminatória. Numa extensa revisão da literatura sobre o impacto do HIV e educação sexual sobre a conduta sexual dos jovens publicada por Unaids (1997), 6 de 15 estudos controlados mostraram ausência de qualquer efeito sobre a atividade sexual. A experiência mostra que mudar uma conduta não é tarefa fácil e, portanto, é preciso saber que nenhum programa de educação em sexualidade terá resultados profundos a curto prazo ${ }^{18-20}$.

Era possível esperar que, entre estudantes de nível universitário que freqüentavam cursos de ciências da saúde, houvesse um nível de conscientização sobre os riscos inerentes às relações sexuais e que a maior parte das sexualmente ativas praticassem sexo seguro. Foi, portanto, decepcionante verificar que a metade delas relatou praticar sexo sem proteger-se adequadamente da possível contaminação por DST. A distribuição das estudantes pesquisadas em Curitiba e Cascavel segundo a conduta sexual vem corroborar discussões realizadas por diversos autores de que o fato de serem universitárias não afeta o comportamento sexual|, 12,13,21-23.

$\mathrm{O}$ aspecto encorajador foi que a prática de sexo seguro foi maior entre aquelas de menor idade, particularmente na análise multivariada. Isto pode ser indicação de que os mais jovens têm mais consciência do risco e de que a tendência a médio prazo pode ser de aumento desta prática segura. É possível, entretanto, que seja apenas indicação de que quanto mais idade, o relacionamento possa ser mais prolongado e estável, e isto leve a um relaxamento no uso do preservativo.

Outro fator que mostrou diferença significativa na conduta sexual assumida foi a pessoa com quem a estudante residia no período da faculdade. Foi interessante verificar que, tanto na análise bivariada como na multivariada, as estudantes que não moravam com a família praticavam sexo seguro em maior proporção que as que moravam com os pais ou parceiro. Este resultado é inverso ao esperado, sugerindo que os pais podem não estar se comunicando adequadamente com suas filhas, e têm dificuldades para conversar sobre o exercício de uma conduta de sexo seguro. Esta diferença pode ser atribuída à possibilidade do menor uso de preservativo com o parceiro com o qual se mora. Mais provável é que as estudantes que moram sozinhas ou com colegas tenham que assumir a responsabilidade pessoal de se cuidar e estabeleçam redes sociais onde a discussão destes assuntos é mais fácil que o que se observa, infelizmente, dentro da casa dos pais.

\section{CONCLUSÃo}

Pode-se concluir que o fato de serem estudantes de nível universitário e freqüentarem cursos das ciências da saúde não é garantia de conduta sexual segura e que, pelo contrario, é evidente que falta ainda muito a se avançar para que estas jovens assumam totalmente a necessidade de se proteger efetivamente contra as DST/ aids. É verdade que o uso de preservativo não é apenas responsabilidade das mulheres, que a colaboração dos homens é indispensável, mas esperava-se que, neste nível de educação, as estudantes tivessem suficiente autonomia para poder negociar com maior eficácia esta proteção com seus parceiros. Tratando-se de futuras profissionais da saúde, deve ser considerado que as jovens destes cursos, além de viverem de modo inadequado sua sexualidade, serão multiplicadoras de ações educativas, necessitando ser, preferencialmente, sadias, bem informadas e livres de preconceitos.

\section{Conflito de interesse: não há.}

\section{SUMMARY}

RISKY SEXUAL BEHAVIOR AMONG UNIVERSITY STUDENTS IN HEALTH SCIENCE COURSES

OBJECTIVE. To evaluate the sexual behavior of female students enrolled in the Department of Health Science of the Federal University of Paraná and the Department of Biological and Health Scienceof the State University of Paraná at the Cascavel campus.

METHODS. All the female students, 18 to 24 years of age, enrolled in the above departments in June 2001 were included in the final sample comprised 572 students in Curitiba and 395 in Cascavel. The study evaluated age, family relationship, religiousness, participation in sex education classes and age at initiation of sexual activity. The use of contraceptive methods and condoms, as well as the number of partners, were variables used to evaluate sexual behavior. Safe sex was defined as the use of a condom by monogamous students in all or in the majority of sexual intercourse and the use of condoms by polygamous students during all sexual intercourse. Unsafe sex was defined as the occasional use of condoms by monogamous students and systematic non-use by polygamous students. The data was 
collected by a self-administered questionnaire. Data analysis was carried out using SPSS and the Pearson and Yates chi-square test, the Wilcoxon-Gehan " $p$ " test, bivariate analysis and logistic regression.

RESULTS. There was no statistically significant difference in the risky sexual behavior between students and the city in which they were studying. Around $50 \%$ of the students aged 18 to 20 and $70 \%$ of those 21 to 24 years of age were sexually active. Abstinence was associated with lower age, greater attendance at religious services and a good relationship between the student and her parents. The practice of safe sex was directly associated with a lower age and living away from home. Participation in sex education classes was associated with greater abstinence but not with safe sex. This relation was not maintained after multivariate analysis.

CONCLUSION. A healthy family relationship and religiousness was associated with safe sexual practices. The percentage of students who still practice unsafe sex is considerable and these results show that being a university student in a health science course does not ensure safe sexual behavior. [Rev Assoc Med Bras 2007; 53(2): I|6-21]

KeY worDS: Sexual behavior. Sexual abstinence. Safe sex.

\section{REFERÊNCIAS}

I. Pinheiro MLE, Moura MCM. Adolescência: sintoma da modernidade. In: O adolescente e a modernidade. Congresso Internacional de Psicanálise e suas Conexões: Companhia de Freud; 2000. p.232-4l.

2. Eisenstein E. Adolescência: definições, conceitos e critérios. Adolescência \& Saúde 2005:2:6-7.

3. Svenson LW, Varnhagen CK. Knowledge, attitudes and behaviours related to AIDS among first-year university students. Can J Public Health. 1990;81:139-40.

4. Short R. Teaching safe sex in school. Int. J Gynecol. Obstet 1998;63: | 47-50

5. Brasil. Ministério da Saúde. Boletim Epidemiológico AIDS. 2002; [acesso em: 19 jan 2003]; I5(I). Disponível em: http://www.aids. gov.br/final/biblioteca/bol marco 2002/tabela3.htm.

6. Paraná. Secretaria de Estad̄o da Saúde. Instituto de Saúde do Paraná. Centro de Informações e Diagnóstico em Saúde. Comitê de Mortalidade Materna; 200I.

7. Fagundes ML, Faria MA, Malc JC. Uso e conhecimento contraceptivo entre estudantes de medicina. Femina. 1993;21:593-01.

8. Szepesvari R, Settergren M, Kasesela S, Mabiriizi D. Sexual life of medical students. East Afr Med. J 1999;76:658.

9. Peltzer K. Factors affecting condom use among south african university students. East Afr Med J. 2000;77:46-52.

I 0. Siegel DM, Klein DI, Roghmann KJ. Sexual behaviour, contraception, and risk among college students. J. Adolesc. Health. 1999; 25:336-43.
I I. Poulson RL, Eppler MA, Satterwhite TN, Wuensch KL, Bass LA. Alcohol consumption, strength of religious beliefs, and risky sexual behaviour in college students. J Am Coll Health. 1998;46:227-328.

12. Mckelvey RS, Webb JA, Baldassar LV, Robinson SM, Riley G. Sex knowledge and sexual attitudes among medical and nursing students. Austr NZJ Psychiatr. 1999;33:260-6.

1 3. De Luca L, Lorençon M, Pasaron AC, Carvalho LR, Ribeiro RA, De Luca HM. Estudo do comportamento psicossocial e biossexual de adolescentes do sexo feminino: mudanças registradas num intervalo de 15 anos. GO Atual. 1988;7:19-27.

I 4. Karofsky PS, Zeng L, Korosok MR. Relationship between adolescentparental communication and initiation of first intercourse by adolescents. J Adolesc Health. 2000;28:4I-5.

| 5. Diaz M. Goodson P. Faúndes A, Aoki F, Pinto e Silva |L. O Risco de contaminação com vírus da AIDS e percepção desse risco entre estudantes da UNICAMP. Reprodução. 1991;6:239-43.

16. Carneiro RM, Ludermir AB, Duarte MSM, Moreira MFA, Campelo DEC, Melo LMP. Comportamento de risco para AIDS entre estudantes universitários: experiência da UFPE. Anais Fac. Med UFPE. 1999;44: | | 3-7.

17. Bosompra K. Determinants of condom use intentions of university students in Ghana: an application of the theory of reasoned action. Soc Sci Med. 2001;52:1057-69.

18. UNAIDS. Impact of HIV and sexual health education on the sexual behaviour of young people: a review update. Geneva: UNAIDS Best Practice Collection; 1997.

19. Kirby D. School-based prevention programs: design, evaluation, and effectiveness. In: DiClemanet RJ, editor. Adolescents and AIDS: a generation in jeopardy. Newbury Park: Sage; 1992.

20. Kassirer A, Griffiths J. The effectiveness of "The Responsible Sexuality Program": a brief high school sexual education intervention. J Sex Educ Ther. 1997;22:5-1।.

2 I. Turner C, Anderson P, Fitzpatrick R, Fowler G, Mayon-White R. Sexual behavior, contraceptive practice and knowledge of AIDS of Oxford university students. J Biosoc Sci. 1988;20:445-51.

22. Bruyn L, Joubert G. The perceptions of female university students regarding STDs and HIV. S Afr Med J. 2002;92:202-3.

23. Caetano ME, Linhares IM, Fonseca AMC, Cardoso EB, Horie MDC, Conceição PSP, et al. Aspectos relacionados ao comportamento sexual e conhecimento sobre doenças sexualmente transmissíveis de um grupo de estudantes da Universidade de São Paulo. Rev Bras Ginecol Obstet. 2004; 15:190-9.

Artigo recebido: 12/04/06

Aceito para publicação: 07/10/06 